\title{
Of Features and Models: A Reflexive Account of Interdisciplinarity across Image Processing, Papyrology, and Trauma Surgery
}

\author{
Ségolène M. Tarte \\ University of Oxford
}

\begin{abstract}
Image processing specialists rarely work on their own, entirely disconnected from the domains of application for which the image processing algorithms are required. In this chapter, I scrutinize my experience of developing image processing approaches for Medicine and for Classics. Through this reflexive take on interdisciplinarity and knowledge exchange and by relating my own experience to the literature on interdisciplinarity, I present observations and strategies that have proven useful in handling the intrinsic difficulties of multidisciplinary collaborative undertakings.

What I call T-words, words that Trigger a Terminology Twitch, are those abstract words that apparently have an obvious meaning, but, within their respective context, are in fact semantic handles that implicitly activate fieldspecific frameworks. Identifying them and investigating their deep meaning in their field-specific context is therefore an essential first step in establishing a working multi-disciplinary collaboration.

Furthering the knowledge exchange process, it is essential to learn about the epistemological foundations of the domains. These are made as much of
\end{abstract}

\section{How to cite this book chapter:}

Tarte, S M. 2016. Of Features and Models: A Reflexive Account of Interdisciplinarity across Image Processing, Papyrology, and Trauma Surgery. In: Bodard, G \& Romanello, M (eds.) Digital Classics Outside the Echo-Chamber: Teaching, Knowledge Exchange \& Public Engagement, Pp. 103-120. London: Ubiquity Press. DOI: http://dx.doi.org/10.5334/bat.g. License: CC-BY 4.0. 
the established conventions of the domain (of collaboration, of publication) as of the actual practices (e.g. familiarity with ways of seeing and looking-in particular when it comes to applying image processing techniques to different fields).

Finally, in response to the black box problem-where algorithms are perceived as producing difficult to interpret output-I contend that experts' minds are black boxes too, and that it is therefore at least as important (if not more) to establish trust between experts as it is to make the black boxes transparent.

\section{Introduction}

Image processing specialists rarely work on their own, entirely disconnected from the domains of application for which the image processing algorithms are required. In this paper, I scrutinize my personal experience as an image processing expert working for applications in the domains of computer-assisted orthopaedic and trauma surgery, and for applications in papyrology and palaeography. It is therefore a reflexive take on interdisciplinarity and knowledge exchange and how the author has experienced them. The assumption is that, although the thoughts laid bare here are drawn from personal experience, many of the working processes and strategies deployed might be generalizable. The intention is not to crystallise a dualistic vision of the sciences and the humanities (as expressed by C.P. Snow ${ }^{1}$ ), but rather to unpick the approaches I have adopted in the very specific contexts of image processing applied for computer-assisted surgery and radiotherapy on one hand, and for papyrology and palaeography on the other hand. I will first introduce these domains of application through the identification of polysemic words that proved key to the research endeavour. Having identified the importance of acknowledging the frameworks that such words implicitly carry with them, I will broaden the perspective by relating my experiences and observations to more general considerations on epistemic cultures, in particular: of modes of knowledge creation and of collaborative models. Finally, I will address the question of methodologies, arguing that as a complement to an understanding of field-specific epistemic cultures, an understanding of the cognitive underpinnings of the research process along with tuned narratives can only benefit collaborative research in Digital Classics and in the Digital Humanities.

\section{Polysemy of Words Crossing the Wires of Communication}

In order to set the scene of interdisciplinary research and the difficulties that can be encountered, I shall first present examples of the use of words in image processing, trauma surgery, papyrology, and palaeography that have, in 
my experience, lead to confusion. These confusions are interesting because, although they might seem anecdotal at first glance, they are symptomatic of a deeper knowledge exchange challenge. The two following words have proven to be polysemic and to carry with them, as implicit luggage, much more than their apparent meaning; they are: 'feature', and 'model.'

\subsection{Where 'Features' are Prominent}

The noun 'feature', according to the Oxford English Dictionary online, when not referring directly to the body or face, is defined as: ${ }^{2}$

[...] (4) A distinctive or characteristic part of a thing; some part which arrests the attention by its conspicuousness or prominence. [...]

In all the academic contexts where I've encountered the word 'feature', the word always takes on this meaning of distinctive or characteristic part, yet each context also appends more to the word and assumes some kind of general, yet field-specific, framework. Within the computer sciences, whilst 'feature' appears both in the image processing expression 'feature detection' and in the pattern recognition and machine learning expression 'feature vector', the features in question do not designate the same abstraction-there can be some overlap, but in general they are rather different objects. In image processing terms, feature detection is the search for specific behaviours of the colours or grey levels in an image (assumed here for simplicity to be a grey scale image), such as sharp changes. These sharp changes can be described quite simply in the pixel-value space as a sudden drop or increase in value when moving from one region to another (steps), but they can also be characterised by a change of behaviour in a transform space, such as the presence of a local maximum in the accumulator space of a Hough transform (used for example to detect lines); in pattern detection, a feature vector is a row of numerical descriptors where each value characterises an aspect of a specific pattern that is searched for, such as Fourier descriptors which have the property of being translation, rotation, and scale invariant, and are therefore quite useful to describe a shape, abstraction made of its position in space (hence a very useful property when one has a template of a shape that is being searched for). After some years working alongside other image processing and pattern recognition experts, this explicit distinction faded, and context naturally and implicitly informed the meaning of the word 'feature'. Further, working specifically in computer-assisted surgery and radiotherapy, the word 'feature' came to mean 'any medically interesting structures' for the surgeons and oncologists I was collaborating with. For example, if the bright white streaks appearing in Computerised Tomography (CT) scans and radiating 
around metallic elements such as screws and plates are sensu stricto features in an image processing context, to medical doctors, those are artefacts, where an artefact is understood as noise that obscures regions of interest and that needs removing. In computer-assisted trauma surgery, the features that surgeons were interested in were all the visual evidence revealing aspects and configurations of the broken bony anatomy; in oncology, the features were all the visual evidence leading to the identification of tumours and their extents. The shift from trauma surgery to oncology meant a readjustment of my understanding of the word 'feature', but only insofar as the structures of interest in the CT images were different structures: in oncology, the specific application was lung cancer; in trauma surgery, it was pelvic and acetabular fractures. Changing the domain of application to work with papyrologists, the word 'feature' was also used, but this time to describe the palaeographical characteristics of the scripts that papyrologists were working on. Whereas in the medical domain, the word 'feature' was a technical word used primarily by image processing experts and adopted by medical doctors to designate the areas of interest in their specific context, in a palaeographical context the word 'feature' is already used routinely to describe a script, its specificities, and the specificities of scribal schools or even hands; what papyrologists and I were facing as collaborators was an encounter of two different pre-existing uses and thus meanings of the word 'feature', not just a sliding of meaning from one domain into another to accommodate needs and work processes. Interestingly, medieval digital palaeographers use the word 'feature' to designate 'A descriptive label which can be applied to a component, idiograph or graph (e.g. "long", "short", "wedged").'3 In this endeavour, an explicit effort is made to produce a definition of the word 'feature' that fits both the contexts of palaeography and of image processing.

Other examples of the use of the word 'feature' in different domains range from archaeology to corpus linguistics, and from electron microscopy to human-computer interaction; what all have in common is that although the meaning of the word is at first glance the same and seems to adhere to the OED definition, each usage actually implicitly carries a contextual framework that relies on skilled vision, on a socially and culturally learned way of looking and seeing that is deeply field specific. ${ }^{4}$

\subsection{Where 'Models' can Fit}

Models and modelling have been of long-standing interest in the Digital Humanities, as demonstrated by a panel discussion at the 2014 Digital Humanities conference, ${ }^{5}$ and by a number of extensive threads on the Humanist Discussion Group. ${ }^{6}$ The object here is not to discuss what modelling in the Digital Humanities might be, but rather to show how speaking of models and 
modelling might differ from field to field. The noun 'model', according to the Oxford English Dictionary online is: ${ }^{7}$

(I) A representation of structure, and related senses.

$[\ldots]$

(I-8-a) A simplified or idealized description or conception of a particular system, situation, or process, often in mathematical terms, that is put forward as a basis for theoretical or empirical understanding, or for calculations, predictions, etc.; a conceptual or mental representation of something. Freq. with modifying word. [...]

As a verb, 'to model' is defined as: ${ }^{8}$

$[\ldots]$

(5-b) To classify, arrange in a system

$[\ldots]$

(10-a) to devise a (usually mathematical) model or simplified description of (a phenomenon, system, etc.)

$[\ldots]$

In all the academic contexts where I've encountered the word 'model', it was used to designate as much the process as the result, and it was used as much to understand a phenomenon or object as to simplify it in order to use it for a specific purpose. In computer-assisted trauma surgery of the pelvis, the noun 'model' was used to designate two distinct objects, one digital, and one physical. The digital object was a 3D virtual model of the broken pelvis, built from CT data of the broken anatomy. This $3 \mathrm{D}$ model allowed surgeons to visualize the $3 \mathrm{D}$ anatomy directly, rather than to have to mentally extract the 3D information from the stack of $2 \mathrm{D}$ images that constitute the CT data. This 3D model extraction, which is now routinely performed, was in its infancy and very much limited by computational power when I was working with surgeons (over 10 years ago). The virtual models of the fragments could further be manipulated independently from one another and abstracted from the soft tissue surrounding them; they allowed surgeons to see the fragments as $3 \mathrm{D}$ objects in their entirety ahead of physically manipulating them in the operating room (OR). ${ }^{9}$ The producing of the virtual object went through the act of modelling, which in this case meant translating the characterisation of the bony anatomy from visual criteria into mathematical image properties. In cases where the pelvic and acetabular fractures are complex, trauma surgeons often resort to physically manipulating a plastic model of the pelvis. On this physical model, they can trace the fracture lines as they understand them from the CT data and from the 3D virtual model, and even recreate the fragments by breaking the plastic model according to the fracture lines; by further referring to the classifications of pelvic and acetabular fractures, ${ }^{10}$ they can decide upon an adapted surgical approach to reduce the fracture. 
In complement to the virtual 3D model, the plastic model is a useful tool not only for teaching, but also for researching and understanding how the pieces of the complex 3D jigsaw puzzle that is the fractured anatomy fit together; ${ }^{11}$ with this physical model, surgeons already engage with an aspect of the surgical act before stepping into the OR.

In lung cancer radiotherapy the model that we ${ }^{12}$ produced was a $4 \mathrm{D}$ model ${ }^{13}$ an animated 3D model, informing oncologists of the most probable location of the tumour at any point within the breathing cycle, thereby helping them to establish a radiotherapy plan-unlike bones, whose geometry is fixed, lungs are flexible and change shape during breathing, so that a tumour within the lungs will move during the breathing cycle, thereby requiring the radiotherapy treatment to take tumour movement into account. The process of building the model revealed many aspects of the variability in breathing, and even if the final model did not take all the variations into account, the process itself informed us of these and allowed us to identify some of the shortcomings of the model (breathing is irregular, especially in lung cancer patients, and the model is by essence regular, even if it allows for statistical deviations). Here again, the process of modelling involved the translation of a real-world phenomenon (breathing, as captured by CT images) into a mathematically formalised behaviour (the $4 \mathrm{D}$ model).

In papyrology, an example of modelling is the work I conducted to simulate the Artemidorus Papyrus as a roll ${ }^{14}$ in order to assess the reordering of the fragments proposed by D’Alessio. ${ }^{15}$ Here again, modelling involved simplification and idealisation as it used the equation of a spiral to describe the roll-and that only will describe a perfect roll, not a skewed roll, no looseness in the roll, no folds, which all could have occurred of course. The virtual model was however helpful and showed conclusively that reordering the fragments was reasonable; and, just as with the virtual and plastic pelvis models, the physical model that I produced by printing the reconstructed papyrus based on the new fragments order served to physically convince the papyrologists by letting them manipulate an avatar ${ }^{16}$ of the papyrus that let them assess the appositeness of the reordering for themselves.

'Model' was a less contentious word than 'feature', as all collaborators expressly wanted a mathematical model of some sort. The core of the work as an image processing scholar however was always to gather sufficient domain-specific information in order to make the models not only relevant but also useful for the experts. Building a model as a simplified or idealized description or conception of a particular system' requires identifying the aspects of the system or phenomenon that need to be encapsulated, represented by the model in order for the domain experts to find some use for the model.

Other examples of words like 'feature' and 'model' would be: 'pattern' (in trauma surgery, in papyrology, in linguistics, in the computer sciences) 'ontology' (in philosophy, in the computer sciences), 'skeleton' (in trauma surgery, in 
image processing, in palaeography), 'process' (in the cognitive sciences, in the computer sciences, in engineering, in anatomy), and even a word like 'science' takes on a different meaning whether used in English or in Latin languages such as French or Spanish (where its meaning covers all forms of academic knowledge, including the Humanities). ${ }^{17}$ It is worth noting further, that whilst researching rigorous definitions of some of the terms above in the respective contexts I have encountered them in, I was often at pains to find a definition; for example, the word 'feature' does not appear in the index of Bischoff's book on Latin palaeography; ${ }^{18}$ and in the index of Gonzalez and Wood's book, commonly known as the image processing bible, the term 'feature' appears only in 'feature selection' and refers the reader to the word 'descriptor. ${ }^{19}$ These conceptual terms seem to be general enough to often not warrant a dedicated fieldspecific definition, and yet their intrinsic meaning shifts subtly away from their general meaning with use in every field.

The intention in pointing out these words is not to unify their use, or to decide normatively of their meaning, but rather to point out a crucial aspect of collaboration. Such words need to be looked out for, discussed, and clarified, so that all involved can grasp what their interlocutors are talking about; in brief, these words ought to Trigger a Terminology Twitch, and I have therefore dubbed them T-words. T-words often designate abstractions that carry an implicit framework with them, and so discussing them will often open the way to fruitful collaboration. Asking seemingly naive questions of and around T-words has always helped me unveil some of the implicit field-specific assumptions and thus facilitated exchanges. Indeed, T-words act as semantic handles within their varied contexts, implicitly activating field-specific theoretical frameworks within which they take on a specialised meaning, a meaning that relates directly to the mode of knowledge creation of the fields that use them.

\section{Modes of Knowledge Creation}

It is no surprise that the T-words evoked above all deal with designating abstractions. As abstractions, they can only be deeply connected to the domain that handles them. Questioning their deep meaning therefore inevitably leads to asking epistemological questions about the domain they emanate from. Some aspects of applied epistemological enquiry are therefore always present in my approaches to collaboration. Such enquiry enables to understand how epistemic cultures differ from field to field, where an epistemic culture is defined as those amalgams of arrangements and mechanisms-bonded through affinity, necessity, and historical coincidence-which, in a given field, make up how we know what we know' ${ }^{20}$ Conducting a field-specific epistemological enquiry when working on a Digital 
Humanities research project, and indeed on any interdisciplinary project, serves multiple purposes:

1. to identify the methodologies that are being mobilised by making them explicit, be they traditional or computational. As a result, it becomes easier to understand the various modes of thinking that scholars engage in; it also helps with the identification, design, and implementation of adapted methods, be they digital or not, be they interdisciplinary or not;

2. to identify the interesting research questions for all fields involved. As a result, it becomes easier to align the expectations of all scholars involved, thus helping to avoid the trap by which scholars in one field might become disengaged due to a lack of interesting/challenging/relevant research question for them in the collaborative project;

3. to identify the implicit collaborative models of each of the fields involved, making people explicitly aware of the different conventions of publication and dissemination that need to be accommodated within a collaborative research project.

\subsection{Field-specific Epistemologies}

According to Becher and Trowler, the nature of knowledge as it is created within academia can be classified as: ${ }^{21}$

- cumulative and atomistic, yielding discovery/explanation, such as mathematics and physics;

- reiterative and holistic, yielding understanding/interpretation, such as anthropology and history;

- purposive and pragmatic, yielding products/techniques, such as software engineering and clinical medicine;

- functional and utilitarian, yielding protocols/procedures, such as law.

This intriguing and potentially controversial classification correlates well however with my limited experience. In computer-assisted surgery and radiotherapy, it became very soon apparent that computer scientists, as well as medical doctors, engage with research questions in a problem-solving mode; the understanding of a given problem might be somewhat different, but scholars approach it indeed in a 'purposive and pragmatic' manner, aiming to yield a product, a technique, a solution. In trauma surgery for example, a research question might be 'How do we assess the accuracy of the reduction of a fracture?'; related questions such as 'what does accuracy mean? In reference to what? To functional rehabilitation? To geometrical congruency?' need to be addressed ahead of the accuracy question, so that each new 
question can securely build upon the previously established answers. The secure aspect is crucial, even if it is primarily so in the scholars' perception and understanding. This makes this approach a foundationalist approach to knowledge creation..$^{22}$ The accent in these fields is on providing answers (and even if identifying research questions is naturally reiterative, the focus remains on the answers, not on how to best formulate the questions). It is even so obvious that answers have to be provided that, in a publication context (e.g. in the computer sciences), a cursory glance at the titles of journal or conference articles will show how it is the methodology and methods used to get the answers that are highlighted-as answers are assumed to be provided. This type of approach might be dubbed diagrammatic, and its main characteristic is that it is predominantly linear, each step requiring the previous one to be completed before build upon it.

In papyrology and palaeography, scholars approach their problems iteratively, which means that they have no qualms about continually revisiting, revising, and reformulating a question. This reiterative approach to research questions is at the core of their knowledge creation process. Answers to such questions matter of course, but here, the accent is on the questions. Even if scholars strive for security in their findings, they are always very conscious that new findings might act as modifiers for pre-existing knowledge; in this sense, their approach is more coherentist than foundationalist. ${ }^{23}$ This also affects the titles of publications, where titles will tend to highlight the themes and results (e.g. in palaeography), leaving the often complicated and multilayered process to get them for the core narrative of the paper. This type of approach might be dubbed radial or fractal, its main characteristic is that it operates predominantly through indexing and cross-referencing to build the scaffold of an argument.

This difference in epistemic cultures became particularly obvious to me at a computational palaeography seminar where one half of the scholars were palaeographers and the other half were computer scientists. ${ }^{24}$ This difference in field-specific modes of knowledge creation manifested itself clearly in a lengthy exchange were computer scientists endeavoured to answer palaeographers' questions by proposing tools and solutions, and upon hearing the answers, palaeographers kept reformulating their questions, refining criteria, evoking exceptions and special cases.

For an image processing expert, it means that in computer-assisted surgery and radiotherapy, the task is to provide medical doctors with definite answers/ tools, whereas with papyrologists and palaeographers, it will never be possible to provide a tool that is a definite answer; the best that the tool can be is a useful way to get elements of an answer that will allow scholars to refine their question in order to create new knowledge. An example of such a tool is the work that Campagnolo et al. have conducted, to create a reference tool for the identification of the types of stains and associated damage that can occur in manuscripts. ${ }^{25}$ 


\subsection{Collaborative Models}

Drawing further on Becher and Trowler, it becomes evident that the collaborative models I have experienced as well as the publication conventions I have encountered correlate with what they call the urban and rural contexts scenarios: $:^{26}$

urban context: characterised by a high people-to-problem ratio, with 'a generally busy-occasionally frenetic-pace of life, high levels of collective activity, close competition for space and resources, and a rapid and heavily used information network. The areas of study are generally narrow, with discrete and separable problems; there are few salient topics; the changes in the research landscape are fast (fast-paced research); competition is intense; and there is also more funding available in urban-type fields;

rural context: characterised by a low people-to-problem ratio, and only displaying the characteristics of urban areas in occasional bursts. The areas of study are wide with open problems that are not sharply delineated; a wide range of themes exists (in contrast to the salient topics of urban contexts); issues are long range, requiring time-demanding research; labour is divided, the lone-scholar model is a frequently found one; and less funding is available in rural-type fields.

When engaging in interdisciplinary research, it is important to know what kind of environment one steps into because the associated collaborative model and publication conventions will reflect elements of the urban/rural context along with elements of the linear/radial thinking and associated epistemologies. There are a number of within-field collaborative models that range from little to no collaboration to huge teams: ${ }^{27}$

- The little-to-no collaboration model is that of the lone scholar, of which a prime example would be St Augustine.

- Small teams will tend to adopt the sports team model, a model where the hierarchy is very flat, and all team members' voices have equal standing.

- Larger teams will tend to adopt an orchestra model, where there is a very clear hierarchical structure and decisions tend to be made from the top.

- The last model, which has recently gained visibility through citizen sciences projects such as those of the Zooniverse suite ${ }^{28}$ (and which is not mentioned in Becher and Trowler's 2001 work), is that of huge teams that rely on a (possibly fluctuating) base of volunteers performing simple tasks that have been designed by researchers having adopted a problem reduction approach in order to gather large amounts of data. ${ }^{29}$ 
For the computer sciences and for medical applications the context is urban, and medium to large teams of researchers work on a project. The teams might however have different kinds of dynamics and ways of organising labour. In the medical domain, the most common model is that of the orchestra. A surgeon for example, habituated to being the decision-maker in the OR, will naturally fall into a conductor role, where all the others in the team (the members of the orchestra) have very precise roles, and the responsibility of coordinating and bringing all the pieces of work together is the conductor/surgeon's. As an image processing expert, stepping into such a collaborative environment means meeting expectations; it does not always allow for being creative and proposing new or different ways of tackling the global problem, so that out-of-the-box thinking is only appreciated if it remains strictly within the confines of one's specific domain of expertise. One possible explanation for this is the amount of pressure and time constraint that surgeons and oncologists are under, which leaves them with little time and patience for what they might perceive as unorthodox thinking. In contrast, the model I've encountered with papyrologists is more one that follows that of a sports team, where all players contribute to an overall task, for example the transcription, edition, and commentary of a papyrus. In my experience, an image processing expert in this context is welcome as an other and different voice.

These organizational differences in how teams operate is clearly reflected in publications, this time through authorship. Single author publications are extremely rare in the computer sciences and in the medical sciences, and when they occur they usually are a sign of seniority. Not only multiple authors are the norm, the order of the authors in the list of authors is meaningful. In the biomedical sciences for instance, the last author is usually the head of department, or senior scholar who received the funding to conduct the work, regardless of the actual amount they have contributed to the work being published. In the Humanities, the norm is more that of single author publications. This poses intriguing questions for Digital Classics, and more generally for the Digital Humanities, whose conventions are still in the process of being developed and might deviate quite significantly from the Humanities tradition of single author publications - as Digital Humanities projects often bring together people from different epistemic cultures. $^{30}$

Having thus reviewed some of the global considerations of interdisciplinary work, tying in my personal experiences and observations with the literature, I now come back to more specific considerations to show how collaborative work has lead me to consider how field-specific narratives of knowledge creation affected my research in computer-assisted trauma surgery and in Digital Classics. 


\section{Tuning Narratives for Knowledge Exchange \& Communication}

\subsection{Cognition for Epistemology}

Citing Becher and Trowler, it is important to attend to 'cognitive as well as social factors in any attempt to make sense of academic interaction. ${ }^{31}$ In trauma surgery, as well is in papyrology, I have found it not only useful but also inspiring to attempt to understand the processes in which experts engage. I have therefore endeavoured to never shy away from asking the candid questions in an attempt to understand how my image processing work might help experts in their work without replacing them. A rule of thumb has been to attempt to grasp what experts are familiar with, in order to ensure that, whilst proposing a new tool, the new tool retains some elements of familiarity, thereby encouraging the uptake of the tool. One example from trauma surgery would be my attempt to transpose and generalise their criteria for the evaluation of the accuracy of fracture reduction. The mode of visualization that trauma surgeons were the most familiar with (over 10 years ago, but this is most likely still the case) was cross-sections of the anatomy so that when I programmed the piece of software that allowed them to visualize and virtually manipulate $3 \mathrm{D}$ virtual models of bone fragments, I made sure to add a functionality that would allow them to see the cross-sectional outlines of the fragments along a plane that they could interactively reposition in space. That was a success, as this cross-sectional visualization chimed with what they were used to looking at when working with CT images. However, drifting away from familiarity, I further attempted to develop a single quantitative measure for the accuracy of the repositioning of the fragments; ${ }^{32}$ to the best of my knowledge, although this measure was a mathematical success, it was never really adopted, I believe that the main reason is that it was too remote from the three measurements that they were used to making to evaluate reduction accuracy.

A striking example of familiarity in papyrology involves more than familiarity and points towards one of the characteristics of experts as having internalised so much of their own cognitive processes that they have built shortcuts making them blind to aspects of what they are actually doing (integration of cognitive units $\left.^{33}\right)$. I was asked to remove the horizontal striae of the woodgrain from an image of a ancient Roman tablet, as they were perceived as a distraction, as noise in the data, which I did. ${ }^{34}$ And although the papyrologists were thankful, I noticed that they made little use of these woodgrain-free images, reverting to using the images with the visible woodgrain. I first thought that the images were too unfamiliar, but it later emerged that although the woodgrain was originally perceived as noise, it had in fact been an implicit source of information; where grooves of the woodgrain were present, it was possible to hypothesize that, for 
instance, the horizontal bar of a ' $\mathrm{T}$ ' might have disappeared in a groove, which the lack of information on the location of the woodgrain precluded. Familiarity is of course only one of the aspects of the cognitive involvement of experts in their work. As evoked earlier when discussing the polysemy of the word 'feature', skilled vision also is a specific form of cognitive involvement. Investigating the types of cognitive involvement of scholars in their research helps make their methodologies explicit by uncovering processes ranging from conceptual to perceptual processes for which the digital world can help provide triggers and support. ${ }^{35}$

Embarking on investigations into the cognitive underpinnings of experts' research processes does not mean that I have become an expert myself however. I argue that one of the crucial aspects of collaborative work resides in how the narratives about specialist research operate.

\subsection{Deep Knowledge and Communicable Knowledge}

Domain-specific knowledge creation requires a layered understanding of fieldspecific concepts; in a collaborative context, experts in a given field need on one hand the deep knowledge that made them experts and on the other hand ways to communicate this deep knowledge in broader brushstrokes so that nonexperts might gain enough of an intuitive understanding of the domain to collaborate fruitfully. One approach to achieving this is by exposing the specialist processes. ${ }^{36}$ Exposing specialist processes fits with the Digital Humanities transparency agenda, ${ }^{37}$ but I contend that this exposing of research processes is intrinsic to the Digital Humanities in a deeper way. Indeed, to engage with, evolve, and create digital tools, it is necessary to understand the underlying methodologies-those same methodologies that are shaped by epistemic cultures and cognitive engagement. My contention is that beyond the transparency agenda, collaborators should not be expected to become experts in a field that is remote from their own. So that more than transparency, what is required is to establish trust between specialists, ${ }^{38}$ and this trust can only be built if experts, and/or some skilled intermediaries, know how to communicate intuitively the essence of expert knowledge and methodologies to non-experts. It is through this fine-tuning of field-specific narratives to non-experts that trust can be established and solid foundations set for fruitful and exciting collaborations. The emergence of the transparency agenda in the Digital Humanities has often been brandished as a solution to what is perceived as the computational black box problem, where the inner working of a black box are hidden, generating anxiety towards the interpretability of the output of the black boxes. But black boxes are not the exclusivity of computational tools, experts' cognitive powers are black boxes too. So more than attempting to make black boxes transparent, I contend that unpicking the epistemic and cognitive underpinning of research 
questions in order to hone interdisciplinary communications between experts will allow us to make methodologies explicit and therefore facilitate the creation of adapted, useful, and trustworthy digital methods.

\section{Conclusions}

Through describing my experiences as an image processing expert for applications in fields that are as different as computer-assisted surgery and papyrology, I have teased out the aspects that I have found to be critical to the success of interdisciplinary research. One of the salient outcomes of this reflexive take is that methodologies have become, of necessity, a central point of enquiry in Digital Classics, and more generally in the Digital Humanities. The drive to build digital tools therefore has incited a reflexive look on methodologies, and I have reviewed herein the various elements that such an approach can shed a light upon. In substance, engaging in epistemic and cognitive enquiries can only facilitate multidirectional cross-pollination and simultaneously well-balanced knowledge exchanges and field-specific knowledge enrichment. Crucial to interdisciplinary collaborations are the following considerations:

1. an acute attention to communication through the identification of what I have called the T-words (those that ought to Trigger a Terminology Twitch) and the naive questions of and around them in order to uncover the implicit theoretical frameworks they carry with them;

2. an identification of the field-specific epistemic cultures, along with their specific epistemologies and collaborative models, in order to clarify expectations and establish balanced research agendas for all involved;

3. a tuning of narratives where cognitive powers and computational tools are not perceived as black boxes anymore, but rather as trustworthy and adapted tools that serve the common research project as much as the field-specific research agendas.

In this sense, by adopting a decidedly cognitive approach to research, and to understanding a given domain of application, cross-disciplinary exchanges can be facilitated. In particular, cognitive approaches to the study of textual artefacts can inform image processing experts who can then propose not just re-purposed approaches, but re-engineered approaches that might themselves be further reengineered to benefit the domain of application they were originally inspired by.

\section{Acknowledgements}

This work has largely benefited from lengthy conversations with the following people, to whom the author is very grateful: Alan Bowman, Pip Willcox, David 
De Roure, Terhi Nurmikko-Fuller, Dominique Stutzmann, Gabriel Bodard and Matteo Romanello, as well as the publisher's reviewers.

\section{Notes}

${ }^{1}$ Snow 1959.

${ }^{2}$ OED s.v. “Feature’: <http://www.oed.com/view/Entry/68848> (last accessed November 2015).

${ }^{3}$ DigiPal, 'Glossary': <http://www.digipal.eu/help/glossary> (last accessed November 2015).

${ }^{4}$ Grasseni 2007; Tarte 2014.

5 Panel 'What is Modeling and What is Not?', chair: Paul Spence, panel members: Van Zundert, JorisJob; Jannidis, Fotis; Drucker, Johanna; Rockwell, Geoffrey; Underwood, Ted; Kestemont, Mike; Andrews, Tara; SperbergMcqueen, Michael; cf. p. 13 <https://dh2014.files.wordpress.com/2014/07/ dh2014-conference-program.pdf $>$ (last accessed November 2015).

${ }^{6}$ cf. for example: Humanist Discussion Group: Vol. 7, No. 0006 (1993); Vol. 16, No. 526 (2003); Vol. 28, No. 74 (2014); on <http://dhhumanist.org/> (last accessed November 2015).

7 OED s.v. "model n.": <http://www.oed.com/view/Entry/120577> (last accessed November 2015).

${ }^{8}$ OED s.v. "model v.": <http://www.oed.com/view/Entry/120578> (last accessed November 2015).

9 Two options were available, simply visualizing the $3 \mathrm{D}$ objects on a $2 \mathrm{D}$ screen, or visualizing them using stereoscopic technology that aimed to facilitate actual 3D perception.

${ }^{10}$ Letournel \& Judet 1993; Tile 1996.

${ }^{11}$ Citak et al. 2008.

${ }^{12}$ In that project, I was part of a team of image processing experts, hence 'we'.

${ }^{13}$ McClelland et al. 2006

14 Tarte 2012.

15 D'Alessio 2009.

${ }^{16}$ I use the term 'avatar' here, where others might have used 'facsimile' or 'surrogate.' 'Avatar' simply underlines that this specific remediation of the artefact only captures some aspect of its materiality, here its affordance to being rolled.

17 The list goes on, of course: 'multimodal', 'record', 'network', 'fieldwork', 'infrastructure', 'agent', etc.

${ }_{18}$ Bischoff et al. 1990.

19 Gonzalez \& Woods 2008.

${ }^{20}$ Knorr Cetina 1999: 1.

${ }^{21}$ Becher \& Trowler 2001: 36.

${ }^{22}$ Haack 1993, chap. 1. 
${ }^{23}$ Haack 1993, chap. 1.

${ }^{24}$ Hassner et al. 2014.

${ }^{25}$ Chap. VII in this volume.

${ }^{26}$ Becher \& Trowler 2001: 106.

27 Becher \& Trowler 2001: 124.

28 Smith et al. 2013.

${ }^{29}$ See also Brusuelas (Chap. X in this volume).

${ }^{30}$ It will not have escaped to the sagacity of astute readers that this very chapter might be considered a Digital Humanities paper and yet follows the single authorship model of the (more traditional) humanities. This chapter is however written in the first person which is a practice more frequently observed in the sciences (and in the English language). In the context of this volume, this autobiographical voice is a deliberate choice: the use of the personal pronouns 'I' and 'we' serves to reinforce the fact that although the observations presented here are likely to apply in many contexts and have the potential to be widely useful, they carry no claim to universality as they are but mediated by the author.

${ }^{31}$ Becher \& Trowler 2001: 124.

32 Tarte et al. 2006.

${ }^{33}$ Feltovich et al. 2006; Chi 2006.

${ }^{34}$ Tarte 2011.

35 Tarte 2014; Terras 2005.

${ }^{36}$ As exemplified by the chapters by Vitale (Chap.VIII), Almas \& Beaulieu (Chap. IX), Bodard \& Stoyanova (Chap. V) in this volume.

37 Ramsay \& Rockwell 2012.

${ }^{38}$ Hassner et al. 2014.

\section{References}

Becher, T. \& Trowler, P. R. (2001). Academic tribes and territories. (2nd Edn.) Buckingham: The Society for Research into Higher Education and Open University Press.

Bischoff, B., Translated by Ó Cróinín, D. \& Ganz, D. (1990). Latin Palaeography - Antiquity of the Middle Ages. Cambridge: Cambridge University Press.

Chi, M. T. H. (2006). Two approaches to the study of experts' characteristics. In Ericsson et al. (Eds.). Chapter 2: 21-30.

Citak, M., Gardner, M., Kendoff, D., Tarte, S., Krettek, C., Nolte, L.-P. \& Hüfner, T. (2008). Virtual 3D planning of acetabular fracture reduction. J Orthop Res, 26(4):547-552.

D’Alessio, G. (2009). On the "Artemidorus" Papyrus. Zeitschrift für Papyrologie und Epigraphik, 171:27-43. 
Ericsson, K. A., Charness, N., Feltovich, P. J., and Hoffman, R. R. (Eds.) (2006). The Cambridge Handbook of Expertise and Expert Performance. Cambridge: Cambridge University Press.

Feltovich, P. J., Prietula, M. J. \& Ericsson, K. A. (2006). Studies of expertise from psychological perspectives. In Ericsson et al. (Eds.). Chapter 4: 41-67.

Gonzalez, R. C. \& Woods, R. E. (2008). Digital Image Processing. (3rd Edn.). Upper Saddle River, NJ: Pearson Education.

Grasseni, C. (Ed.) (2007). Skilled visions: between apprenticeship and standards, volume 6 of EASA - Learning Fields. New York: Berghahn Books.

Haack, S. (1993). Evidence and Inquiry: towards reconstruction in epistemology. Oxford: Blackwell.

Hassner, T., Sablatnig, R., Stutzmann, D. \& Tarte, S. (2014). Digital Palaeography: New Machines and Old Texts (Dagstuhl Seminar 14302). Dagstuhl Reports, 4(7):112-134.

Knorr Cetina, K. (1999). Epistemic Cultures. How the Sciences make knowledge. Cambridge, Massachusetts: Harvard University Press.

Letournel, E. \& Judet, R. (1993). Fractures of the acetabulum. (2nd Edn.). Heidelberg, Germany: Springer-Verlag.

McClelland, J., Blackall, J., Tarte, S., Chandler, A., Hughes, S., Ahmad, S., Landau, D. \& Hawkes, D. (2006). A continuous 4D motion model from multiple respiratory cycles for use in lung radiotherapy. Med Phys, 33(9):3348-3358.

Smith, A. M., Lynn, S. \& Lintott, C. J. (2013). An introduction to the zooniverse. In First AAAI Conference on Human Computation and Crowdsourcing, volume AAAI Technical Report CR-13-01.

Snow, C. P. (1959). The Two Cultures. (Canto Edn. 1993). Cambridge: Cambridge University Press.

Ramsay S. \& Rockwell G. (2012) Developing Things: Notes towards an Epistemology of Building in the Digital Humanities. In M. K. Gold (Ed.), Debates in the Digital Humanities. Minneapolis: University of Minnesota Press.

Tarte, S. (2014). Interpreting textual artefacts: Cognitive insights into expert practices. In Mills, C., Pidd, M., and Ward, E. (Eds.), Proceedings of the Digital Humanities Congress 2012, Studies in the Digital Humanities. Sheffield: HRI Online Publications.

Tarte, S., Talib, H., Ballester, M. G. \& Langlotz, F. (2006). Evaluating partial surface matching for fracture reduction assessment. In Biomedical Imaging: From Nano to Macro, 2006. ISBI 2006. 3rd IEEE International Symposium on, pages $514-7$.

Tarte, S. M. (2011). Papyrological investigations: Transferring perception and interpretation into the digital world. Lit Linguist Computing, 26(2): $233-47$. 
Tarte, S. M. (2012). The digital existence of words and pictures: The case of the Artemidorus papyrus. Historia, 61(3):325-336 (+bibliog. pp 357-61; fig. pp 363-5).

Terras M. (2005). Reading the readers: Modelling complex humanities processes to build cognitive systems. Lit Linguist Computing 20:41-59.

Tile, M. (1996). Acute pelvic fractures: I. causation and classification. Journal of the American Academy of Orthopaedic Surgeons, 4(3):143-151. 